Draft VERSION JunE 1, 2020

Preprint typeset using LATEX style AASTeX6 v. 1.0

\title{
AEROSOLS AND WATER ICE IN JUPITER'S STRATOSPHERE FROM UV-NIR GROUND-BASED OBSERVATIONS
}

\author{
MAnuel López-Puertas \\ Instituto de Astrofísica de Andalucía (CSIC), Glorieta de la Astronomía s/n, E-18008 Granada, Spain \\ Pilar Montañés-Rodríguez ${ }^{1}$, E. Pallé ${ }^{1}$ \\ Instituto de Astrofísica de Canarias, C/Vía Láctea s/n, E-38200 La Laguna, Spain
}

M. HÖPFNER

Institute of Meteorology and Climate Research, Karlsruhe Institute of Technology, Karlsruhe, Germany

A. SÁnchez-López, M. García-Comas and B. Funke

Instituto de Astrofísica de Andalucía (CSIC), Glorieta de la Astronomía s/n, E-18008 Granada, Spain

${ }^{1}$ Departamento de Astrofísica, Universidad de La Laguna, Av., Astrofísico Francisco Sánchez, s/n, E-38206 La Laguna, Spain

\begin{abstract}
Jupiter's atmosphere has been sounded in transmission from the UV to the IR, as if it were a transiting exoplanet, by observing Ganymede while passing through Jupiter's shadow. The spectra show strong extinction due to the presence of aerosols and haze in Jupiter's atmosphere and strong absorption features of methane. Here, we report a new detailed analysis of these observations, with special emphasis on the retrievals of the vertical distribution of the aerosols and their sizes, and the properties and distribution of the stratospheric water ice. Our analysis suggests the presence of aerosols near the equator in the altitude range of $100 \mathrm{hPa}$ up to at least $0.01 \mathrm{hPa}$, with a layer of small particles (mean radius of $0.1 \mu \mathrm{m}$ ) in the upper part (above $0.1 \mathrm{hPa}$ ), an intermediate layer of aerosols with a radius of $0.3 \mu \mathrm{m}$, extending between $\sim 10$ and $0.01 \mathrm{hPa}$; and a layer with larger sizes of $\sim 0.6 \mu \mathrm{m}$ at approximately $100-1 \mathrm{hPa}$. The corresponding loads for each layer are $\sim 2 \times 10^{-7} \mathrm{~g} \mathrm{~cm}^{-2}, \sim 3.4 \times 10^{-7} \mathrm{~g} \mathrm{~cm}^{-2}$, and $\sim 1.5 \times 10^{-6} \mathrm{~g} \mathrm{~cm}^{-2}$, respectively, with a total load of $\sim 2.0 \times 10^{-6} \mathrm{~g} \mathrm{~cm}^{-2}$. The lower and middle layers agree well with previous measurements; but the finer particles of $0.1 \mu \mathrm{m}$ above $0.01 \mathrm{hPa}$ have not been reported before. The spectra also show two broad features near 1.5 and $2.0 \mu \mathrm{m}$ which we attribute to a layer of very small $(\sim 10 \mathrm{~nm}) \mathrm{H}_{2} \mathrm{O}$ crystalline ice in Jupiter's lower stratosphere $(\sim 0.5 \mathrm{hPa})$. While these spectral signatures seem to be unequivocally attributable to crystalline water ice, they require a large amount of water ice to explain the strong absorption features.
\end{abstract}

Keywords: planets and satellites: atmospheres, planets and satellites: composition, planets and satellites: gaseous planets

\section{INTRODUCTION}

The study of our solar system as a template for exo-planetary systems is a very useful and obvious first-step approach for exploring and learning about new worlds (Christensen \& Pearl 1997; Turnbull et al. 2006; Robinson et al. 2011; Seager 2014). In particular, mimicking exoplanet transits by observing eclipses in our solar system is a useful resource when no remote sensing platforms are available to do the measurements. Recent examples for the Earth and for Jupiter atmospheres have been recently carried out by Palle et al. (2009) and Montañés-Rodríguez et al. (2015), respectively. 
Those observations, in addition of providing insights for future exoplanet characterizations, also serve for exploring in details our planetary atmospheres themselves, as is the case of this work. Understanding aerosols in Jupiter's atmosphere is very important since, among other major effects, they largely influence the energy balance of the middle atmosphere (West et al. 2004; Zhang et al. 2015). A very good review of the work carried until 2004 is given by West et al. (2004). More recently, several works have been done on retrieving their latitudinal and vertical distributions in the troposphere and stratosphere (see, e.g., Kedziora-Chudczer \& Bailey 2011; Zhang et al. 2013, 2015). However, the detailed composition and structure of the stratospheric haze remains uncertain, largely because of the lack of the necessary data. Moreover, except in our previous work of Montañés-Rodríguez et al. (2015), the presence of water ice in Jupiter's stratosphere has not been reported so far. The study of the properties and amounts of water ice is necessary for understanding Jupiter' stratosphere.

Here we report on the limb transmission spectra of Jupiter's atmosphere obtained by using ground-based observations of Ganymede, which is in synchronous rotation around Jupiter, when crossing Jupiter's shadow. During the eclipse, the spectral features of the Jovian atmosphere are imprinted in the sunlight that, after passing through Jupiter's planetary limb, is reflected from Ganymede toward the Earth (see Fig. 1 in Montañés-Rodríguez et al. 2015). The ratio spectrum of Ganymede before and during the eclipse removes the spectral features of the Sun, of the local telluric atmosphere on top of the telescopes, and the spectral albedo of Ganymede. The spectra cover the range from the UV to the near-IR and have a high spectral resolution ( $\mathrm{R}=17,400$ for the VIS and 11,300 for the near-IR) and high signal-to-noise ratio. Previous results of the analysis of the spectra by Montañés-Rodríguez et al. (2015) include the simulations of the $\mathrm{CH}_{4}$ absorption bands, the attribution of the 1.5 and $2.0 \mu \mathrm{m}$ absorption features to $\mathrm{H}_{2} \mathrm{O}$ ice, as well as the detection of the atomic transitions of Na.

Here we focus on a more detailed analysis of the VIS and near-IR spectral regions. While in the previous study we analysed only the early umbra spectrum here we re-analysed that spectrum and also examine the penumbra and the mid-umbra spectra. This allows us to conduct a detailed study of the haze in Jupiter's stratosphere. Thus, the retrievals of the vertical distribution and sizes of the aerosols are new. In addition, we have studied the effects of the altitude distribution of the water ice layer, the ice particle size and their temperature. The revised study has also led to a revision of the water ice content in the stratospheric water ice layer, which is about a factor of 5 larger.

In the next two sections we describe the observations and the details of the geometry, radiative transfer model and input data required to analyze the spectra. In Sec. 4 we discuss the different components that contribute to the transmission spectra and several sensitivity studies, and in Sec. 5 the results obtained for the distributions and sizes of the aerosols and of the water ice. Then, we discuss the results and present the conclusions in Secs. 6 and 7, respectively.

\section{OBSERVATIONS}

An eclipse of Ganymede was first observed on 06/10/2012 using LIRIS (Manchado et al. 2004) at WHT in La Palma Observatory, Spain. The experiment was later repeated by observing a second eclipse with XSHOOTER (Vernet et al. 2011) at VLT in Paranal Observatory, on 18/11/2012. This work focuses on the analysis of VLT data which, given the larger aperture of VLT, have a larger signal to noise ratio. WHT observations, however, exhibit essentially the same spectral features. A detailed description of the observations can be found in Montañés-Rodríguez et al. (2015).

One of the major advantages of these data is that the observations were taken simultaneously in a broad spectral range, covering from 0.3 to $2.5 \mu \mathrm{m}$, and at a high spectral resolution, with resolving powers of $\mathrm{R}=9100,17400$ and 11300 for the UV, VIS and NIR regions, respectively. The typical observing window for this kind of eclipse is only several minutes. Hence, spectra of Ganymede were taken continuously in stare mode along its translation through Jupiter's shadow. The penumbra phase started at 04:19 UT and the umbra phase at 04:40 UT. A total of 41 individual spectra, covering the whole spectral region, where taken for Ganymede before the eclipse, 8 during the penumbra, and 3 during the umbra. From those measurements, penumbra and umbra transmission spectra were obtained by normalizing them (calculation of the ratio) with a few of the spectra measured just before the eclipse started. In this way, the ratio of the spectra cancels out the telluric contribution of the atmosphere, the solar spectral features and the spectral signatures of Ganymede. As shown by Montañés-Rodríguez et al. (2015) (see their Fig. 2), the airmass change (decrease) that occurs during the eclipse did not have a significant effect on the penumbra and umbra normalized spectra at wavelengths longer than $0.5 \mu \mathrm{m}$, the region studied in this work.

\section{OBSERVATIONAL GEOMETRY, RADIATIVE TRANSFER MODEL AND INPUT PARAMETERS}

Transmission spectra of the occultation of the Sun through Jupiter's atmosphere as observed from Ganymede have been simulated for the observations of the eclipse in the penumbra and within the first stages of the umbra. The effect of the strong refraction of Jupiter's atmosphere allows for the limb sounding of different altitudes of its atmosphere as 
the Sun sets from Ganymede. Thus, the penumbra and umbra spectra measured during the eclipse provides us with information on different regions of Jupiter's stratosphere. This is illustrated in Fig. 1 which shows the normalized geometric contribution of the Sun's disk at different phases during the penumbra and umbra. We can see that at some phases of the eclipse, the projected Sun's disk (as viewed from Ganymede) on Jupiter's limb can be as low as a few tens of $\mathrm{km}$. The larger value denotes the Sun's centre, and the sounded altitudes extend above and below. We should also note the asymmetry of the sounded region, wider above and narrower below the Sun's centre, caused by the weaker (stronger) refraction of the thinner (denser) atmosphere above (below) the Sun's centre. We should also note that the Sun's disk projected on Jupiter is less than 2 degrees in latitude and hence we are then sensing only 1 or 2 degrees of the Jupiter equatorial zone. The consideration of the refraction effects for sounding different atmospheric layers has also been recently illustrated by Dalba et al. (2015) in the analysis of a Cassini/VIMS solar occultation of Saturn.

The transmission spectra have been calculated by using the Karlsruhe Optimized and Precise Radiative Transfer Algorithm (KOPRA, Stiller et al. 2002). KOPRA is a well-validated line-by-line radiative transfer model which has implemented all the necessary physics for studying this problem. The code was originally developed for its application to Earth's atmosphere studies and has been lately adapted to the atmospheres of Titan and Mars (García-Comas et al. 2011; Robert et al. 2012), and now for Jupiter.

Our simulations include, besides the $\mathrm{CH}_{4}$ absorption bands, Rayleigh scattering by molecular hydrogen and helium, collisions induced absorption (CIA) for $\mathrm{H}_{2}-\mathrm{H}_{2}$ and $\mathrm{H}_{2}-\mathrm{He}$, and Mie scattering by aerosols and water ice.

Rayleigh scattering by molecular hydrogen and helium, which provides significant opacity mainly in the ultraviolet, have been taken into account by including the Rayleigh optical cross sections provided by Ford \& Browne (1973) for $\mathrm{H}_{2}$ and of Chan \& Dalgarno (1965) for helium. Its effect on the spectral range studied here of 0.5-2.5 $\mu \mathrm{m}$ is, however, of little significance compared to the other absorptions (see the cyan line in Fig. 3 at wavelengths of 0.5-0.7 $\mu \mathrm{m}$ ).

Ro-vibrational absorption bands resulting from collisions between pairs of $\mathrm{H}_{2}-\mathrm{H}_{2}$ and $\mathrm{H}_{2}$ - $\mathrm{He}$, the so-called collisions induced absorption or CIA, are significant only in Jupiter's lower atmosphere, where they form a smooth feature centered in the 2.0-2.5 $\mu \mathrm{m}$ spectral region. Our simulations include this feature with absorption coefficients at low temperatures, derived by Borysow (2002) for $\mathrm{H}_{2}-\mathrm{H}_{2}$ pairs, and by Borysow et al. (1989) and Borysow \& Frommhold (1989) for $\mathrm{H}_{2}$-He.

Other gases such as $\mathrm{CO}_{2}, \mathrm{CO}$ and $\mathrm{H}_{2} \mathrm{O}$ were considered, but because of their very low concentrations did not contribute significantly to the absorption spectra. $\mathrm{NH}_{3}$ in the gas phase was found to absorb significantly only at the low troposphere where the spectra are saturated at most wavelengths by the $\mathrm{CH}_{4}$ bands. Thus, it was not included. The molecular spectroscopic data for all species have been taken from the HITRAN compilation, 2012 edition (Rothman et al. 2013).

In addition to the absorption due to the gaseous species, the Rayleigh scattering and the CIA absorption, we also considered the Mie scattering by two kinds of stratospheric aerosols. One of them is constituted of aerosol particles similar to those derived by Zhang et al. (2013) for equatorial regions from ground-based NIR spectra and multiplephase-angle images from the Cassini Imaging Science Subsystem (ISS). The other is water ice (see below).

In our derivation of aerosol information from the measured spectra (concentration, altitude distribution and size) we have assumed fixed optical properties. There are several studies in literature about the complex refractive indices of Jupiter's aerosol near the equator although they are still rather uncertain. Mishchenko (1990) derived the real part of the refraction index of the aerosols in the upper troposphere equatorial region of Jupiter with a value of $n_{r}=1.39 \pm 0.01$. Khare et al. (1984) measured that index for tholins-like aerosols in the laboratory obtaining values from 1.62 to 1.70 for the $0.5-2.5 \mu \mathrm{m}$ spectral range. They were measured, however, in an $\mathrm{N}_{2}$ environment, like in Titan, rather than for the $\mathrm{H}_{2}$ environment of Jupiter. On the other hand, the real part of the refractive index of the $\mathrm{NH}_{3}$ ice (Martonchik et al. 1984) has been generally used in the studies of Saturn's upper tropospheric haze (see, e.g. Fletcher et al. 2011; Barstow et al. 2016). This index is very close to 1.4 in the near-IR spectral range, practically the same as that derived by Mishchenko (1990). In this work we have used the $\mathrm{NH}_{3}$ ice $n_{r}$ values measured by Martonchik et al. (1984) for the aerosols and have carried out a test with the higher values and different spectral dependency of the tholins-like aerosols of Khare et al. (1984). The imaginary part of the refractive index of the haze was adjusted to the values derived by Zhang et al. (2013) of 0.02 and $10^{-3}$ at 0.25 and $0.9 \mu \mathrm{m}$, respectively, then, linearly decreasing it to $10^{-4}$ at $1.5 \mu \mathrm{m}$, and keeping it constant at $10^{-4}$ up to $2.5 \mu \mathrm{m}$. As shown below, the imaginary part of the refractive index of aerosols contributes, however, very little to our extinction calculations.

In order to reproduce the absorption features near 1.5 and $2.0 \mu \mathrm{m}$ of the observed spectra (see Fig. 3), we also included in the simulations extinction by water ice assuming Mie scattering. The optical properties of water ice (real and imaginary part of the refractive index) included in the nominal simulations were taken from Mastrapa et al. (2008) for crystalline water ice at the temperature of $150 \mathrm{~K}$, typical of Jupiter's lower stratosphere. However, we also 
performed other tests using the properties for the water ice at temperatures of 200-250 K reported by Warren \& Brandt (2008).

\section{TEST MODEL AND SENSITIVITY STUDIES}

Before carrying out the best fit analysis (see Sect. 5), we performed an analysis of three spectra corresponding to different times of the eclipse - penumbra, early umbra, and mid umbra- which sound different altitudes of Jupiter' stratosphere. Thus, we started from a basic test model and carried out sensitive studies that allowed us to explore and roughly confine the parameters space that contribute to the transmission spectra. We should note that this model is slightly different from the best-fit model for this spectrum obtained after performing the retrieval from the three spectra (see Sect. 5).

The reference atmosphere of Jupiter used in the model, including pressure, temperature, $\mathrm{CH}_{4}$ (see Fig. 2) and other species abundances, was taken from González et al. (2011). The $\mathrm{CH}_{4}$ vmr profile at the studied altitudes is very similar to those obtained by other models (Moses et al. 2005; Kim et al. 2014), although it is significantly smaller at higher altitudes. Tests using the higher $\mathrm{CH}_{4}$ vmr profile of Moses et al. (2005) (see Fig. 2), and by decreasing the kinetic temperature in $10 \mathrm{~K}$ have been performed (see discussion below).

From previous studies, e.g., Zhang et al. (2013) and by inspection of the transmission spectra (see Fig. 3), it is clear that aerosols contribute to the transmission. Thus, we assume in the test model an aerosol layer that extents from $50 \mathrm{mb}(\sim 70 \mathrm{~km}$ above the $1000 \mathrm{mb}$ level $)$ up to about $0.01 \mathrm{mb}(\sim 300 \mathrm{~km})$ which is mainly concentrated at the lowest altitudes (see Fig. 2). That is, it decays exponentially from a concentration of 4 particles $/ \mathrm{cm}^{3}$ at $50 \mathrm{mb}$ to 0.04 particles $/ \mathrm{cm}^{3}$ near $0.01 \mathrm{mb}$ with a total column density of $2.1 \times 10^{7}$ particles $/ \mathrm{cm}^{2}$. The aerosol particles were assumed to have a mono-modal lognormal distribution with a mean radius of $0.25 \mu \mathrm{m}$ and a distribution width of $\sigma=0.3$. Several tests assuming a bi-modal distribution with different sizes are discussed below. The shape of this lognormal distribution is similar to that of the two-parameters gamma distribution used by Zhang et al. (2013).

The presence of water ice in Jupiter's lower atmosphere was evident from the absorbing peaks near 1.5 and $2.0 \mu \mathrm{m}$. We then included in the test model a thin layer of water ice particles located at the pressure range of 0.5-0.1 mbar (180-200 km; see Fig. 2). The water ice cloud in this model was assumed to be composed of a mono-modal lognormal distribution of small particles, a mean radius of $10 \mathrm{~nm}$, and a distribution width of $\sigma=0.3$, with a peak concentration at the base of the cloud of $3.3 \times 10^{7}$ particles $\mathrm{cm}^{-3}$. This results in a total column number density of $4.3 \times 10^{13}$ particles $\mathrm{cm}^{-2}$.

Figure 3 shows the early-umbra spectrum with simulations including the different absorbers/scatterers of the test model described above. The contributing atmospheric layers to this spectrum correspond to the red line in Fig. 1. The observed and simulated spectra show the most prominent $\mathrm{CH}_{4}$ bands, the extinction of the aerosol particles, and the two distinct absorption features of water ice at 1.5 and $2.0 \mu \mathrm{m}$. The cyan line includes absorption by $\mathrm{CH}_{4}$ bands and also the Collision Induced Absorption (CIA) and the Rayleigh scattering. The latter is very small compared to the other contributions, only noticeable at wavelengths near $0.5-0.7 \mu \mathrm{m}$ at the tangent heights covered by this spectrum (see Fig. 1). The aerosols extinction increases significantly at shorter wavelengths, becoming the dominant component. Water ice presents the two typical broad peaks near 1.5 and $2.0 \mu \mathrm{m}$ but also with significant contributions in the $0.5-1.0 \mu \mathrm{m}$ region and near $2.3-2.5 \mu \mathrm{m}$. With those absorbers, all major features of the measured spectrum are reproduced although the overestimation of the transmission and its different shape in the $0.5-1.0 \mu \mathrm{m}$ spectral region are noticeable.

\subsection{Sensitivity studies}

In order to better understand the extinction features in the measured spectra and to further derive more precise information of the aerosol and water ice layers, we performed several sensitivity studies.

Figure 4 shows the optical depth of the aerosols and water ice for a nadir (vertical) view of Jupiter's atmosphere calculated with the aerosol and water ice profiles of the test model (see Fig.2). That figure shows the relative contribution of the extinction from the haze and the water ice particles at different wavelengths. The haze dominates up to about $1.9 \mu \mathrm{m}$ although water ice has a comparable absorption near $1.5 \mu \mathrm{m}$. Note that this is for the test model described above, and their relative contributions depend on the size distribution of both. We can also see that the haze's absorption (imaginary part) is negligible at all wavelengths studied here. The transmission of aerosols (see Fig. 3) shows a large decrease (strong extinction) at wavelengths shorter than $\sim 1.5 \mu \mathrm{m}$. Thus suggesting that the aerosol's extinction of the test model for the early umbra spectra at $\lambda<1 \mu \mathrm{m}$ is overestimated and does not have the right shape. Figure 4 shows that the water ice extinction (given its assumed characteristics described above) is dominated by absorption (imaginary part) at $\lambda>1.4 \mu \mathrm{m}$ and by scattering extinction at shorter wavelengths. This 
behavior depends on the size of the water ice particles, as discussed below.

The dependency of the aerosol's extinction on its particle's size and its effect on transmission are shown in Fig. 5 . To show more clearly the dependency of the extinction cross section with wavelength, we have scaled them such that the total aerosol volume is conserved, instead of the number of particles. The effects of the aerosol particles size on the transmission of the early umbra spectrum is shown in the bottom panel of Fig. 5. The total column of the particles of the test model has been kept fixed. Here we see that the spectral dependency of the haze transmission is rather sensitive to the particles size. Thus, a way to decrease the extinction at wavelengths smaller than $\sim 1 \mu \mathrm{m}$ in the test model (see Fig. 3) without reducing the aerosol mass load, is to assume that the particles are smaller than the test radius of $0.25 \mu \mathrm{m}$.

Regarding the water ice, we have analyzed several aspects: its temperature dependence, the size of the water ice particles, and the altitude distribution of its concentration. As shown above, the extinction in the spectra are dominated by water ice absorption near 1.5 and $2.0 \mu \mathrm{m}$ (see Fig. 4). The imaginary part of the water ice refraction index, $k$, has been measured in the laboratory under different conditions. Mastrapa et al. (2008) have measured the $k$ index for the crystalline ice at a temperature of $150 \mathrm{~K}$ in this spectral range. Also Warren \& Brandt (2008) has measured it for the ice at the higher temperatures of 200-250 K. These measurements are shown in the top panel of Fig. 6. The transmissions for these two measurements of the water ice are shown in the bottom panel of Fig. 6 for the early-umbra spectrum and the water ice concentration of the test model (see Fig. 2). It is clearly seen that the crystalline ice at a temperature of $150 \mathrm{~K}$ (Mastrapa et al. 2008) yields a better fit at the peaks of the ice absorption bands near 1.5 and 2.0 micron, while for the ice at a temperature of 200-250 K (Warren \& Brandt 2008) the peaks are shifted to shorter wavelengths. Then we conclude that the water ice signatures revealed in the observed spectrum are more likely due to the water ice at a temperature close to $150 \mathrm{~K}$, a typical temperature of the Jupiter stratosphere, rather than at a higher temperature.

Figure 7 shows the sensitivity of the transmission to the water ice particles' size. The upper panel shows the extinction cross-section of the ice particles with the two components: absorption and scattering. Note that the cross sections of particles with mean radii of $r_{m}=0.02-0.05 \mu \mathrm{m}$ have been scaled to the volume of the particles with a mean radius of $0.01 \mu \mathrm{m}$. The figure demonstrates the change of the relative contribution of the absorption peaks with respect to the scattering as the particle's size varies. For small particles, around $0.01 \mu \mathrm{m}$, the absorption peaks near 1.5 and $2.0 \mu \mathrm{m}$ are significantly larger that the scattering contribution while, as the particles grow to about $0.05 \mu \mathrm{m}$, their relative contributions are significantly smaller. The effects on the simulated spectra for the early umbra conditions (red curve in Fig. 1) are shown in the lower panel. The simulations assume the same total volume (or mass) of the water ice, i.e., by adjusting the particles concentration for their sizes, and considering the test model inputs. Here we observe that: i) the extinction is larger at all wavelengths for the larger particles; ii) the slope and shape at the shorter wavelengths of $0.5-1.4 \mu \mathrm{m}$ change dramatically with the particle's size; and iii) the depth of the absorption peaks and the asymmetry in absorption at each side of the peak change significantly. For the smaller particles $(0.01-0.02 \mu \mathrm{m})$ the depth is larger and the absorption is more symmetric, opposite to the absorption of the larger particles $(0.03-0.05 \mu \mathrm{m})$. Hence, the size of water ice particles is likely in the $0.01-0.02 \mu \mathrm{m}$ range.

\section{DERIVED AEROSOLS AND WATER ICE DISTRIBUTIONS}

After the sensitivity study has been done and we establish the likely range of the main parameters, we have performed a best-fit of the three observed spectra for the early phase of the penumbra, the early phase of the umbra and the mid-umbra, respectively. The best-fit was based on obtaining the best balance between the smaller $\chi^{2}$ and the larger correlation coefficient.

Figure 8 shows best-fit transmission spectra for the three measured spectra in the $0.5-2.5 \mu \mathrm{m}$ region. The estimated sounded altitude ranges of Jupiter's atmosphere for these spectra are shown in Fig. 1, corresponding to the black, red, and dark blue lines, respectively. The $\chi^{2}$ is generally very small, with values of $\chi^{2} / N$ in the range of of $6 \times 10^{-5}$ to $1.4 \times 10^{-3}$. The correlation coefficient is very close to one, better for the penumbra spectrum and smaller for the mid-umbra conditions. The obtained distributions of the aerosols and water ice concentrations are discussed below and shown in Fig. 11.

\subsection{Upper region: the penumbra spectrum}

The top curves in Fig. 8 correspond to the higher sounded altitudes of Jupiter stratosphere (see Fig. 1), above $200 \mathrm{~km}(0.2 \mathrm{hPa})$. This measured spectrum does not show any sign of significant water ice absorption, thus indicating that the water ice cloud is below this altitude. In addition to the $\mathrm{CH}_{4}$ absorbing bands, we see significant extinction at $\lambda \lesssim 1.5 \mu \mathrm{m}$ that increases to shorter wavelengths. This indicates the presence of aerosols at high altitudes, above 
$200 \mathrm{~km}(\sim 0.2 \mathrm{hPa})$. Note that the altitude (pressure) registration is rather accurate since the absorption by the $\mathrm{CH}_{4}$ bands, near 1.15, 1.7 and $2.3 \mu \mathrm{m}$ are well reproduced. These altitudes are well above those derived by Zhang et al. (2013) near the equator (see their Fig. 3), comprised in the pressure range of 20-70 hPa. Our observations, measuring through the limb, where the geometry offers a larger optical path, are more sensitive than the nadir-like measurements used by those authors; thus allowing us to measure the highest-altitude aerosols.

The shape of the extinction in the $0.5-1.5 \mu \mathrm{m}$ region also gives us information about the size of these high-altitude aerosols. As shown in Fig. 5, the smaller aerosols produce a steeper transmission. The best fit to this spectrum was obtained when considering two size distributions. The first one, producing most of the absorption at the shorter wavelengths, has a mean radius of $0.10 \mu \mathrm{m}$ and a concentration of 2.66 particles $\mathrm{cm}^{-3}$, extending from $200 \mathrm{~km}$ up to $320 \mathrm{~km}$, giving a column density of $\sim 3.2 \times 10^{7}$ particles $\mathrm{cm}^{-2}$ and an aerosol load, assuming a mass density of about 1 $\mathrm{g} \mathrm{cm}^{-3}$, of $\sim 2 \times 10^{-7} \mathrm{~g} \mathrm{~cm}^{-2}$. In order to fit the spectrum in the $1-2 \mu \mathrm{m}$ region, a less-abundant second distribution of aerosols with a larger radius is required. Hence, the higher altitudes of the $\sim 0.3 \mu \mathrm{m}$ distribution (see Fig. 11) slightly contributes to this spectrum (see Fig. 1). That distribution is the major contributor to the extinction of the early-umbra spectrum (see next subsection) and, in fact, the best fit to the penumbra spectrum alone requires that the concentration in the upper few kms of the $\sim 0.3 \mu$ m-distribution be reduced by a factor of 0.36 .

At these altitudes, however, we see no evidence of the extinction of the $0.6 \mu \mathrm{m}$ larger particles.

\subsection{Middle region: the early-umbra spectrum}

The fitting of the early umbra spectrum is not as simple as for the penumbra spectrum. First, we see clearly the signatures of water ice near 1.5 and $2.0 \mu \mathrm{m}$, although it also contributes at other wavelengths (see Fig. 7). We already discussed that the spectral positions of the peaks near 1.5 and $2.0 \mu \mathrm{m}$ reveal the presence of crystalline water ice at a temperature close to $150 \mathrm{~K}$. Also, from the pronounced absorption valleys near those wavelengths and the very similar absorptions at each side of the valleys (and among the valleys themselves), we deduce very small water ice particles (see Fig. 7). Specifically, from the fit to the early-umbra spectrum in Fig. 8 we derive ice particles with a mean radius of $\sim 0.01 \mu \mathrm{m}$. However, we cannot discard that a fraction of the particles have a larger size with mean radius of $0.02 \mu \mathrm{m}$.

The ice concentration for the best fit (see Fig. 11) is very similar to that of the test model, with a peak value at the base of the cloud of $3.6 \times 10^{7}$ particles $\mathrm{cm}^{-3}$ resulting in a water ice column density of $\sim 4.7 \times 10^{13}$ particles cm ${ }^{-2}$; that is, an ice mass load of $\sim 3 \times 10^{-4} \mathrm{~g} \mathrm{~cm}^{-2}$.

The absorption near the ice peak at $1.5 \mu \mathrm{m}$ has not been fitted very well. We think it is due to a deficiency in our model to adequately reproduce the aerosol slope extinction rather than that of water ice. Note that the depth of the valley is well reproduced; it is the background continuum that actually differs.

As discussed above, the water ice cloud should be located below $\sim 200 \mathrm{~km}(\sim 0.2 \mathrm{hPa})$. On the other hand, given the sounding altitudes of this spectrum (red line in Fig. 1), it has to be present above $100 \mathrm{~km}(\sim 10 \mathrm{hPa})$. We have performed calculations by varying the position of the cloud (between 100 and $200 \mathrm{~km}$ ) and its thickness and have obtained the best fit with a layer of about $30 \mathrm{~km}$ thick with its basis located near $170 \mathrm{~km}(\sim 0.5 \mathrm{hPa})$. Fig. 9 shows two examples where the cloud was compressed to $10 \mathrm{~km}$ and shifted down to $100 \mathrm{~km}$. We clearly see that the ice signatures are worse reproduced and that locating the cloud at lower altitudes results in very weak absorption.

The fitting of the aerosol extinction in this wide spectral range is difficult. Attempts to reproduce the transmission spectra with only one size distribution $(\sigma=0.3)$ and varying the particles mean radii between 0.3 and $0.6 \mu$ m were not satisfactory (see, e.g. Fig. 3). In order to obtain a better fit to the shape at shorter wavelengths but producing a significant extinction at longer wavelengths it was necessary to consider three particle size distributions. In addition to the distribution at $0.3 \mu \mathrm{m}$, one of smaller particles with a mean radius of $0.1 \mu \mathrm{m}$ (see above), producing the right shape at shorter wavelengths, and another of larger particles, mean radius of $0.6 \mu \mathrm{m}$, accounting for the larger extinction at $\lambda>1.5 \mu \mathrm{m}$. The fit shown in the middle curves of Fig. 8 was obtained with a concentration of 0.135 particles cm $\mathrm{cm}^{-3}$ with a mean radius of $0.3 \mu \mathrm{m}$, extending from $80 \mathrm{~km}$ up to $230 \mathrm{~km}$ (see Fig. 11), giving a column density of $\sim 2 \times 10^{6}$ particles $\mathrm{cm}^{-2}$ and an aerosol load of $\sim 3.4 \times 10^{-7} \mathrm{~g} \mathrm{~cm}^{-2}$. Both the $0.1 \mu \mathrm{m}$ distribution discussed above and the $0.6 \mu \mathrm{m}$ layer discussed below also contribute to this spectrum but in order to achieve the best fit they need to be decreased by factors of 0.61 and 0.67 , respectively. Other additional size-intermediate aerosol distributions, and slightly different concentrations (although with very similar mass loads), are not discarded. Small concentrations of particles larger than the distribution of mean radius of $0.6 \mu \mathrm{m}$ with a $\sigma$ of 0.3 are not discarded either.

The extinction at wavelengths of $2.35-2.5 \mu \mathrm{m}$ is significantly underestimated for any aerosol and water ice distributions. The $\mathrm{CH}_{4}$ absorption is rather weak at these wavelengths. It is unclear whether the required excess extinction is caused by imperfect knowledge of the water ice at these wavelengths or any other type of aerosols.

The absorption in the $\mathrm{CH}_{4}$ bands near 1.15, 1.7 and $2.3 \mu \mathrm{m}$ are slightly overestimated for this early-umbra spectrum 
(see Fig. 8). That is, the $\mathrm{CH}_{4}$ number density is overestimated. The $\mathrm{CH}_{4}$ vmr profile we used in the simulations is already in the low limit of the currently accepted profiles (see Fig. 2). Hence, it is unlikely to be caused by the used $\mathrm{CH}_{4}$ vmr. However, the total number density might be overestimated at these altitudes. Simultaneous measurements of the pressure-temperature (p-T) are not available and might differ from the model of González et al. (2011) used here. A colder temperature profile at the lower altitudes would imply a more compressed atmosphere and then lighter at higher altitudes. We have then performed calculations by decreasing the p-T profile in the entire altitude range by $10 \mathrm{~K}$ (see Fig. 2, dash-red line). The resulting transmittances (see Fig. 10) are in better agreement with the calculations, particularly, at the stronger absorptions in the Q-branches of the $\mathrm{CH}_{4}$ bands.

\subsection{Lower region: mid-umbra spectrum}

The mid-umbra spectrum (bottom curves in Fig. 8) is noisier that the other spectra. We even see signatures of emission lines (near $1.05 \mu \mathrm{m}$ ) which reflects such a larger noise. The extinction is stronger and the shape at $\lambda<1 \mu \mathrm{m}$ is significantly different. The simulations suggest that to fit such spectra we need particles larger than $0.3 \mu \mathrm{m}$. The fit in the figure was achieved with a concentration of 0.135 particles $\mathrm{cm}^{-3}$ with a mean radius of $0.6 \mu \mathrm{m}$, extending from $60 \mathrm{~km}$ to $140 \mathrm{~km}$ (see Fig. 11), giving a column density of $\sim 1.1 \times 10^{6}$ particles $\mathrm{cm}^{-2}$ and an aerosol load of $\sim 1.5 \times 10^{-6}$ $\mathrm{g} \mathrm{cm}^{-2}$. The $0.3 \mu \mathrm{m}$ aerosol layer discussed above also contributes to this extinction although in order to obtain the best fit it was slightly (a factor of 0.85 ) attenuated. The water ice layer, however, has to be significantly reduced by a factor of 0.035 . One possible reason for the weaker water ice absorption at the lower altitudes could be that it is not uniformly distributed in clouds.

\section{DISCUSSION}

The mean radius of the aerosol particles derived in this work $(0.1-0.6 \mu \mathrm{m})$ covers the range of 0.2 to $0.5 \mu \mathrm{m}$ derived by Zhang et al. (2013) for the equatorial particles. Our penumbra spectrum shows, however, that the haze distribution of small particles $(0.1 \mu \mathrm{m}$ ) at rather high altitudes (from 0.1 to 0.01 or $0.001 \mathrm{hPa}$ ) is present, otherwise its shape in the $0.5-1.5 \mu \mathrm{m}$ cannot be reproduced. Similarly, the strong extinction, particularly at $\lambda>1.5 \mu \mathrm{m}$, as well as the flatness of the mid-umbra spectrum suggest the presence of large particles (up $0.6 \mu \mathrm{m}$ ). The total load of the haze particles above $100 \mathrm{hPa}$ of $\sim 2.0 \times 10^{-6} \mathrm{~g} \mathrm{~cm}^{-2}$ is very similar to that derived by Zhang et al. (2013) of $\sim 1-2.0 \times 10^{-6} \mathrm{~g} \mathrm{~cm}^{-2}$ at low latitudes (see their Fig. 11). These results are also consistent with the Galileo observations reported by West et al. (2004). The haze layer derived by Zhang et al. (2013) at low latitudes extends, at most, up to about $20 \mathrm{hPa}$. This roughly coincides with our larger-particles haze layer which has a similar mass load of $\sim 1.5 \times 10^{-6} \mathrm{~g} \mathrm{~cm}^{-2}$. However, we need, in addition, the $\sim 0.3 \mu \mathrm{m}$ layer extending up to about $0.1 \mathrm{hPa}$ and even a layer of finer particles $(\sim 0.1 \mu \mathrm{m})$ at higher altitudes. Since our observations use a limb geometry, it is possible that the high-altitude particles could not be resolved by the nadir ground-based NIR measurements and the Cassini-ISS images used by Zhang et al. (2013). We should mention that because our haze layers extends over a wider pressure range than that of Zhang et al. (2013) (at the equator) but have similar mass loading, our concentrations are significantly lower than the ones of those authors. However, they are very similar to those derived by Rages et al. (1999), also taken from a limb geometry.

About the nature of the haze particles, our data do not provide information about what are they made of. Possibly we are observing the compact sub-micron (CSM) particles described by Zhang et al. (2013). The origin of these low latitudes particles is unclear although it has been suggested that it is different from that of the aerosols at mid and high latitudes (Zhang et al. 2013). The latter are assumed to be fractal particles aggregated from very small $(\sim 10 \mathrm{~nm})$ monomers, generated by complex hydrocarbon synthesis driven by the energetic particle precipitation (charged particles) in the auroral region at high latitudes (Wong et al. 2003; Zhang et al. 2013). The low altitude particles measured here, however, seem to be generated via neutral photochemical processes driven by the UV photons (Zhang et al. 2013).

It is worth noting that we overestimate the aerosol extinction in the 1.4-1.7 $\mu \mathrm{m}$ spectral region. We could not adjust the aerosol contribution in the complete $0.5-2.3 \mu \mathrm{m}$ by changing the size or concentrations of the aerosols particles. We used for the real part of the refraction index of the aerosols the values measured by (Martonchik et al. 1984) for the $\mathrm{NH}_{3}$ ice (see Sec. 3). One possible reason is that this index is overestimated in that spectral region. On the other hand, we systematically underestimate the absorption at $2.35-2.5 \mu \mathrm{m}$ (see early umbra spectrum in Fig. 8). It is possible that another aerosol is contributing in this spectral region.

About the water ice layer, the absorption spectral features at 1.5 and $2.0 \mu \mathrm{m}$ can be very well reproduced in our model with crystalline water ice at a temperature of $150 \mathrm{~K}$. The large depths of those features below the aerosol continuum inform us that the particles are very small, of around $10 \mathrm{~nm}$. Larger particles lead to shallower valleys. Thus, these ice particles have a similar size to the monomers that compose the fractal aggregates (West \& Smith 1991) derived by 
Zhang et al. (2013) at high latitudes in Jupiter's stratosphere. The water ice layer is located at pressures below the $0.1 \mathrm{hPa}$ pressure level; otherwise those signatures would also appear in the upper penumbra spectrum. Also, the best fit to the spectra is obtained for a narrow layer which should not extend below $\sim 0.5 \mathrm{hPa}$. The measurements suggest a vertical column of about $4.7 \times 10^{13}$ particles $/ \mathrm{cm}^{2}$ of $\sim 10 \mathrm{~nm}$ size, which implies a water ice mass load of $\sim 3 \times 10^{-4}$ $\mathrm{g} \mathrm{cm}^{-2}$. This mass load is large when compared with current knowledge of the water vapor concentrations in Jupiter's stratosphere. On other hand, this water ice content is about a factor of 5 larger than the previous value obtained in Montañés-Rodríguez et al. (2015). The reason is that we have now performed a better fit to the early umbra spectrum and have included more accurate information of the aerosols absorption derived from the three spectra.

Cavalié et al. (2013) reported from HERSCHEL measurements, a water vapour column density above $\sim 2 \mathrm{hPa}$ of $\sim \times 10^{15} \mathrm{~mol} \mathrm{~cm}^{-2}$ or $1.5 \times 10^{-7} \mathrm{~g} \mathrm{~cm}^{-2}$. That is about 2000 times smaller than the water molecules contained in our derived water ice layer. Considering the gas-ice equilibrium phase, the presence of water ice at the pressure-temperature of this Jupiter region lead to a $\mathrm{H}_{2} \mathrm{O}$ vapour volume mixing ratio of $\sim 0.5$ ppmv. This is five to fifty times larger than the $\mathrm{H}_{2} \mathrm{O}$ volume mixing ratios in the range of $0.01-0.1 \mathrm{ppmv}$ of the Interplanetary Dust Particles (IDP) model and the SL9 comet profiles that were derived by Cavalié et al. (2013) from HIFI/Herschel measurements.

We do not have a clear explanation for such larger amount of water ice. We should mention, however, that the mid-umbra spectra suggests water ice amounts 30 times smaller, which could be explained if the water ice layer is not homogeneously distributed over all longitudes around Jupiter's stratospheric equator. About the origin of this water ice, it seems unlikely that it comes from upper layers. One possibility could be its injection in the lower stratosphere by plumes arising from the troposphere, e.g. as those observed by Sanchez-Lavega et al. (1991, 2008). These plumes are powered by moist water convection and hence might inject significant water contents in the lower stratosphere. The major difficulty of the transport from below is to cross the cold tropopause but, once this is reached, the pressure-temperature conditions in the lower stratosphere are favorable for maintaining water in the form of ice.

\section{SUMMARY AND CONCLUSIONS}

In this work we have analyzed the limb transmission spectra of Jupiter's atmosphere obtained by observing Ganymede from the ground while passing through Jupiter's shadow. The ratio of the spectra of Ganymede before and during the eclipse removes the spectral features of the Sun, most of the telluric absorption and the spectral albedo of Ganymede. The spectra cover from UV to near-IR ( 0.5 to $2.5 \mu \mathrm{m}$ are analyzed here) and have a high spectral resolution and high signal-to-noise ratio. The spectra show strong extinction due to the presence of aerosols in the Jupiter's atmosphere and strong absorption features of methane. Furthermore, the transmission spectra reveal two broad features near 1.5 and $2.0 \mu \mathrm{m}$ that we identified as caused by a water ice layer.

The significant refraction of Jupiter's atmosphere together with the limb geometry allows us to obtain vertical information of Jupiter's stratosphere. In particular we have analysed three transmission spectra: penumbra, early umbra and mid-umbra, that correspond to the altitude ranges of approximately $170-500 \mathrm{~km} ; 80-300 \mathrm{~km}$, and $60-$ $200 \mathrm{~km}$, and have derived a vertical distribution of the aerosols concentrations and some estimations of their size. Specifically, our observations suggest the presence of aerosols near the equator in the altitude range of $100 \mathrm{hPa}$ up to at least $0.01 \mathrm{hPa}$ (this upper limit could not be constrained and it might be even higher), with a clear distribution of a layer of small aerosols with a mean radius of $0.1 \mu \mathrm{m}$ in the upper part (above $0.1 \mathrm{hPa}$ ), an intermediate layer of aerosols with a mean radius of $0.3 \mu \mathrm{m}$ that extend approximately between 10 and $0.01 \mathrm{hPa}$; and a layer of larger aerosols, of $\sim 0.6 \mu \mathrm{m}$ at approximately $100-1 \mathrm{hPa}$. The corresponding loads for each layer are $\sim 2 \times 10^{-7} \mathrm{~g} \mathrm{~cm}^{-2}, \sim 3.4 \times 10^{-7} \mathrm{~g} \mathrm{~cm}^{-2}$, and $\sim 1.5 \times 10^{-6} \mathrm{~g} \mathrm{~cm}^{-2}$, respectively, with a total load of $\sim 2.0 \times 10^{-6} \mathrm{~g} \mathrm{~cm}^{-2}$. While the layer distributions are not well defined, e.g., distributions of intermediate similar sizes can also explain the spectra, the total load is well constrained, as it is the location of the smaller aerosols at the top of the layers and that of the larger ones at the bottom.

The mass load of aerosols reported here is very similar to that derived by Zhang et al. (2013) at low latitudes from ground-based NIR measurements and from the Cassini-ISS images. They are also consistent with the Galileo observations reported by West et al. (2004). Our haze layers comprised between $100 \mathrm{hPa}$ and $20 \mathrm{hPa}$ roughly coincide with those derived by Zhang et al. (2013) at low latitudes, although our particles are larger with two layers of mean radii of 0.3 and $0.6 \mu \mathrm{m}$ while the mean radius of Zhang et al. is $0.3 \mu \mathrm{m}$. However, we also observe an additional layer of finer particles (mean radius of $0.1 \mu \mathrm{m}$ ) above $0.1 \mathrm{hPa}$.

In addition, the spectra reveal the presence of a layer of very small crystalline water ice particles $(\sim 10 \mathrm{~nm})$ at pressure levels of $0.5-0.1 \mathrm{hPa}$, with a mass load of $\sim 3 \times 10^{-4} \mathrm{~g} \mathrm{~cm}^{-2}$. This mass load is much larger (about a factor of 2000) than the column above $2 \mathrm{hPa}$ measured by Herschel (Cavalié et al. 2013). Moreover, the gas-ice equilibrium phase at the pressure-temperatures near $0.5 \mathrm{hPa}$, implies a water vapor concentration of $0.5 \mathrm{ppmv}$, which is a few tens larger than that derived by (Cavalié et al. 2013) in the middle stratosphere. The origin of such large water ice amounts is 
not clear. The weaker water ice absorption at the middle-umbra spectrum suggests, however, that this layer might not be distributed homogeneously along all longitudes. More measurements are required to disentangle whether the large water ice amounts correspond to isolated, or time variable clouds, or if they originate from regular and uniform water ice layers.

We are very grateful to Rafael Escribano, Víctor Herrero, Anni Määttänen, Beatriz Mate, Agustín Sánchez-Lavega, and Miguel Ángel Satorre for very valuable discussions on the water ice topic. The IAA team was supported by the Spanish MICINN under projects ESP2014-54362-P, ESP2017-87143-R, and EC FEDER funds. This work is also partly financed by the Spanish Ministry of Economics and Competitiveness through grant ESP2013-48391-C4-2-R. MGC is also supported by the MINECO under its 'Ramón y Cajal' subprogram.

Facility: XSHOOTER, VLT, Paranal Observatory.

Software: KOPRA, IDL

\section{REFERENCES}

Barstow, J. K., Irwin, P. G. J., Fletcher, L. N., Giles, R. S., \& Merlet, C. 2016, Icarus, 271, 400

Borysow, A. 2002, Astronomy and Astrophysics, 390, 779

Borysow, A., \& Frommhold, L. 1989, ApJ, 341, 549

Borysow, A., Frommhold, L., \& Moraldi, M. 1989, Astrophysical Journal, 336, 495

Cavalié, T., Feuchtgruber, H., Lellouch, E., et al. 2013, Astronomy and Astrophysics, 553, A21

Chan, Y. M., \& Dalgarno, A. 1965, Proceedings of the Physical Society, 85, 227

Christensen, P. R., \& Pearl, J. C. 1997, Journal of Geophysical Research, 102, 10875

Dalba, P. A., Muirhead, P. S., Fortney, J. J., et al. 2015, The Astrophysical Journal, 814, 1

Fletcher, L. N., Baines, K. H., Momary, T. W., et al. 2011, Icarus, 214, 510

Ford, A. L., \& Browne, J. C. 1973, Atomic Data and Nuclear Data Tables, 5, 305

García-Comas, M., López-Puertas, M., Funke, B., et al. 2011, Icarus, 214, 571

González, A., Hartogh, P., \& Lara, L. M. 2011, in Planetary Science (World Scientific Publishing Co. Pte. Ltd.), 209-218

Kedziora-Chudczer, L., \& Bailey, J. 2011, Monthly Notices of the Royal Astronomical Society, 414, 1483

Khare, B. N., Sagan, C., Arakawa, E. T., et al. 1984, Icarus, 60, 127

Kim, S. J., Sim, C. K., Sohn, M. R., \& Moses, J. I. 2014, Icarus, 237, 42

Manchado, A., Barreto, M., Acosta-Pulido, J., et al. 2004, Proc. SPIE, 5492, 1094

Martonchik, J. V., Orton, G. S., \& Appleby, J. F. 1984, Applied Optics, 23, 541

Mastrapa, R. M., Bernstein, M. P., Sandford, S. A., et al. 2008, Icarus, 197, 307

Mishchenko, M. I. 1990, Icarus, 84, 296

Montañés-Rodríguez, P., González-Merino, B., Palle, E., López-Puertas, M., \& García-Melendo, E. 2015, Astrophys J Lett, 801, L8
Moses, J. I., Fouchet, T., Bezard, B., et al. 2005, Journal of Geophysical Research, 110, E08001

Palle, E., Osorio, M. R. Z., Barrena, R., Montañés-Rodríguez, P., \& Martín, E. L. 2009, Nature, 459, 814

Rages, K., Beebe, R., \& Senske, D. 1999, Icarus, 139, 211

Robert, S., Vandaele, A. C., Willame, Y., et al. 2012, in COSPAR Abstract Book, 353

Robinson, T. D., Meadows, V. S., Crisp, D., et al. 2011, Astrobiology, 11, 393

Rothman, L. S., Gordon, I. E., Babikov, Y., et al. 2013, Journal of Quantitative Spectroscopy and Radiative Transfer, 130, 4

Sanchez-Lavega, A., Miyazaki, I., Parker, D., Laques, P., \& Lecacheux, J. 1991, Icarus, 94, 92

Sanchez-Lavega, A., Orton, G. S., Hueso, R., et al. 2008, Nature, 451, 437

Seager, S. 2014, Proceedings of the National Academy of Sciences, 111, 12634

Stiller, G. P., von Clarmann, T., Funke, B., et al. 2002, J. Quant. Spectrosc. Radiat. Transfer, 72, 249

Turnbull, M. C., Traub, W. A., Jucks, K. W., et al. 2006, ApJ, 644,551

Vernet, J., Dekker, H., D’Odorico, S., et al. 2011, A\&A, 536, A105

Warren, S. G., \& Brandt, R. E. 2008, Journal of Geophysical Research, 113, D14220

West, R. A., Baines, K. H., Friedson, A. J., et al. 2004, Jovian clouds and haze, ed. F. Bagenal, T. E. Dowling, \& W. B. McKinnon (Cambridge University Press, UK), 79-104

West, R. A., \& Smith, P. H. 1991, Icarus, 90, 330

Wong, A.-S., Yung, Y. L., \& Friedson, A. J. 2003, Geophysical Research Letters, 30, doi:10.1029/2002GL016661

Zhang, X., West, R. A., Banfield, D., \& Yung, Y. L. 2013, Icarus, 226, 159

Zhang, X., West, R. A., Irwin, P. G. J., Nixon, C. A., \& Yung, Y. L. 2015, Nature Communications, 6, 1 


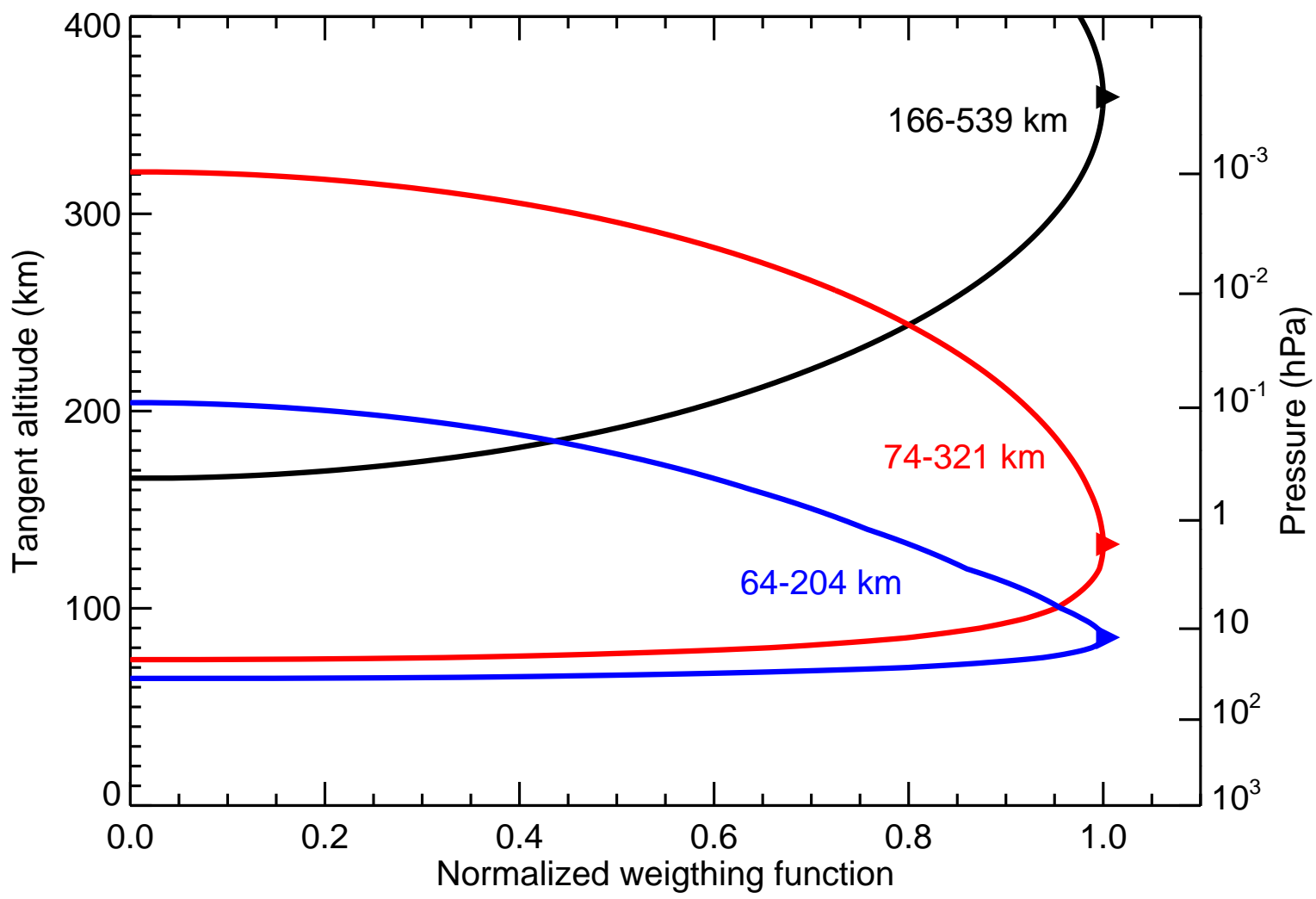

Figure 1. The estimated tangent altitudes covered by our measured penumbra (black), early umbra (red), and mid-umbra (blue) spectra. 


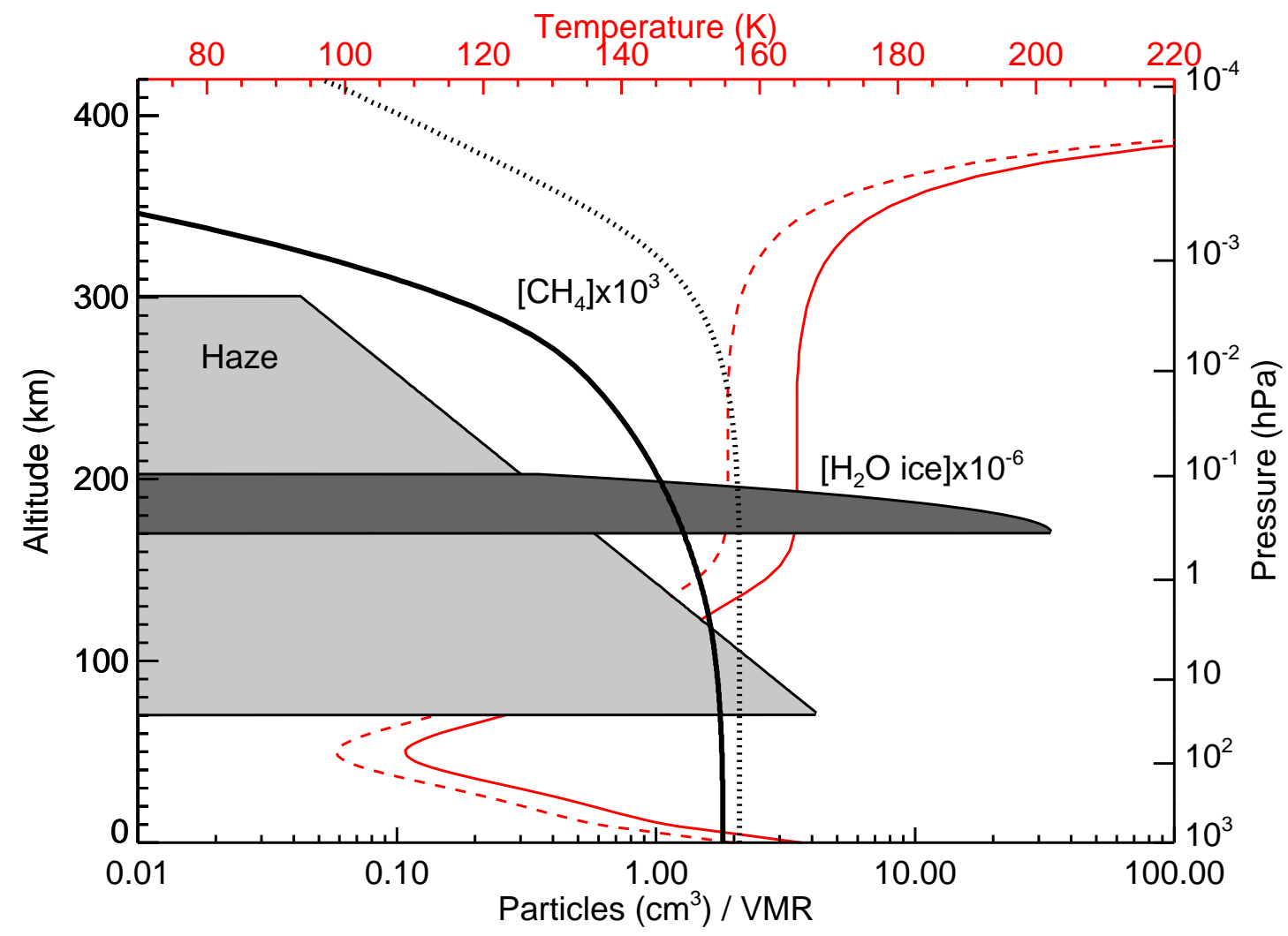

Figure 2. The distribution of haze (aerosols), $\mathrm{CH}_{4}$, and $\mathrm{H}_{2} \mathrm{O}$ ice used in the test model of the transmission spectra (see Fig. 3). Altitude is taken as zero at the 1 bar pressure level. The $\mathrm{CH}_{4}$ vmr profiles correspond to those of González et al. (2011) (solid, nominal model) and of Moses et al. (2005) (dotted). The nominal temperature profile (solid) and reduced by $10 \mathrm{~K}$ (dashed) are also shown in red (upper scale). 


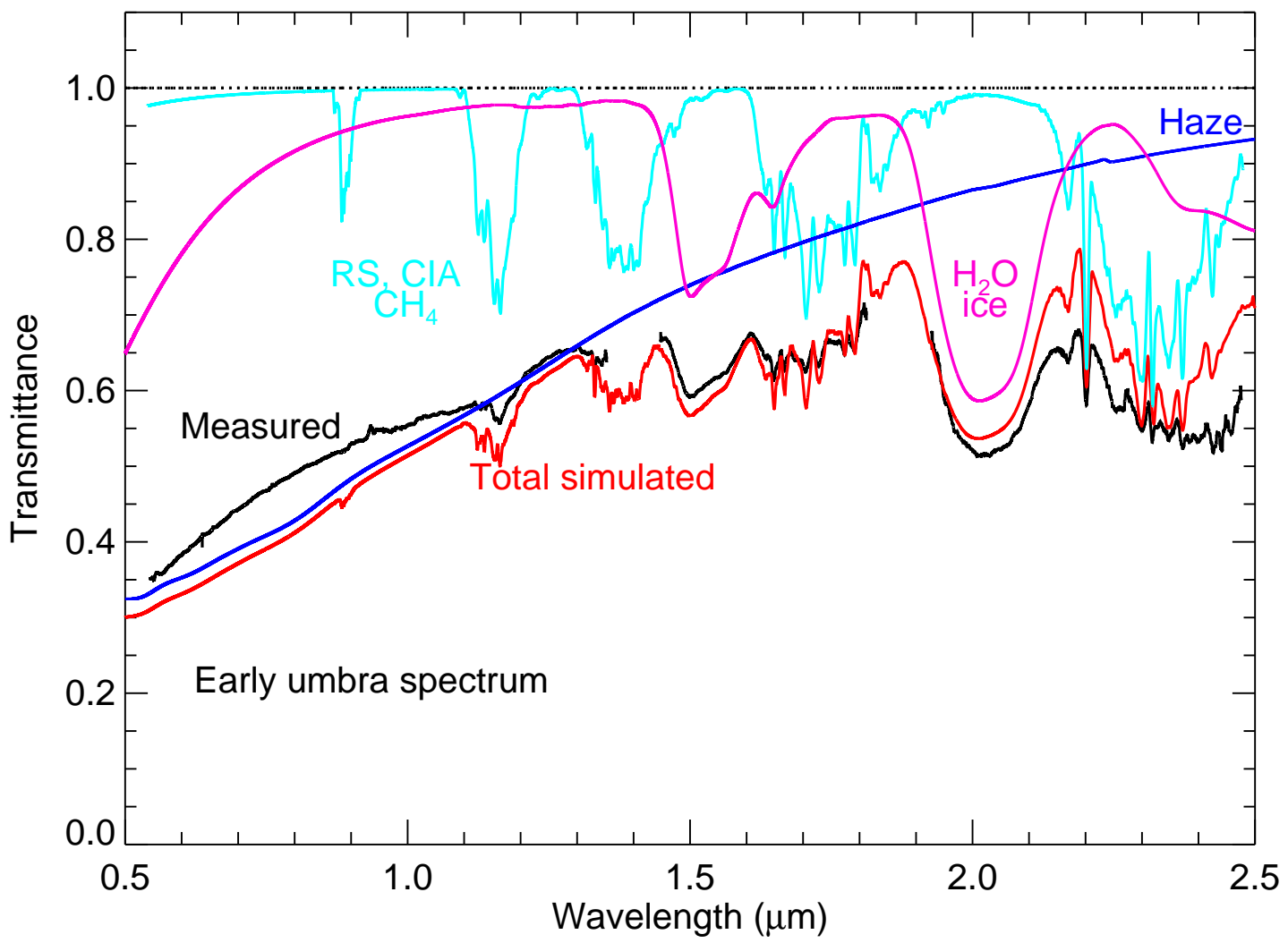

Figure 3. Contributions of the $\mathrm{CH}_{4}$, Rayleigh scattering and CIA absorption (in cyan), aerosols (haze) (in dark blue), and the $\mathrm{H}_{2} \mathrm{O}$ ice (in magenta) to the extinction of the early umbra spectrum. Measured spectrum in black and simulated spectrum in red.

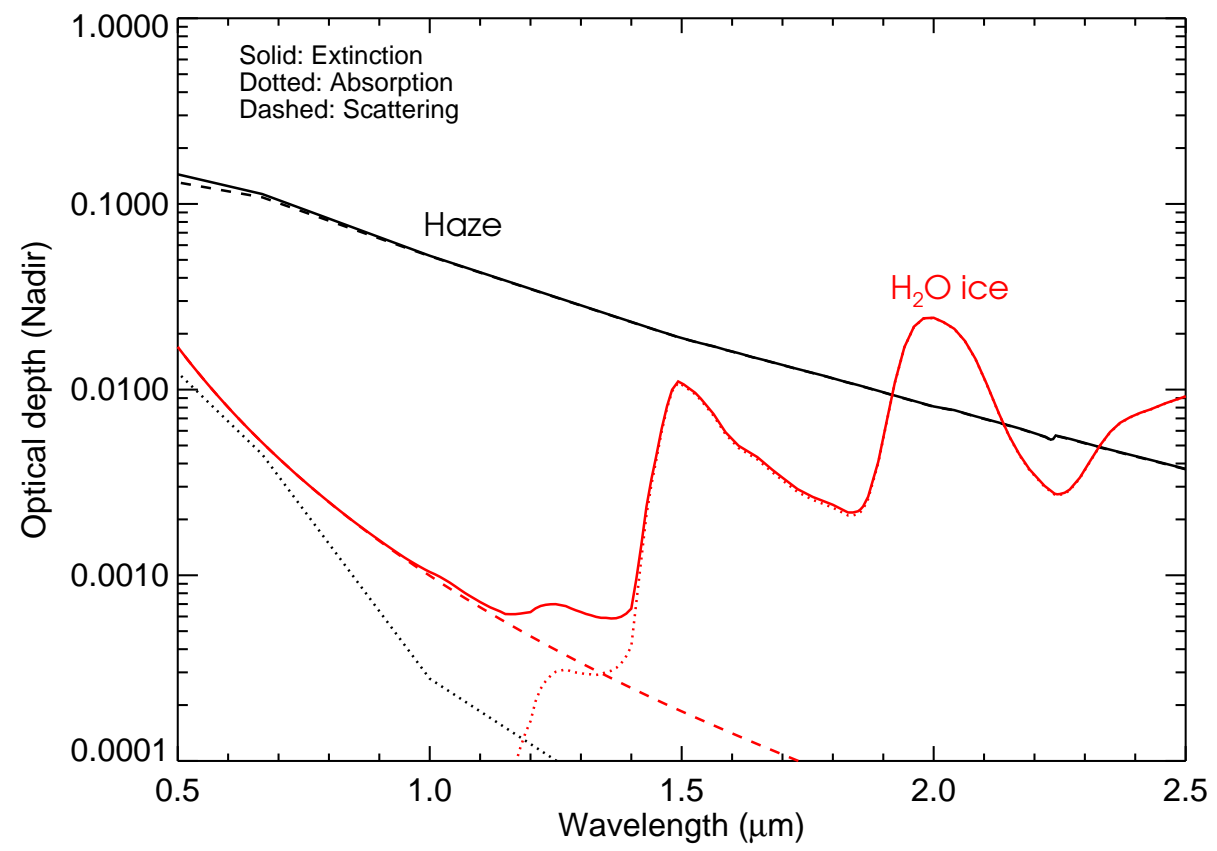

Figure 4. Optical depths of the aerosols and water ice for a Jupiter nadir (vertical) geometry computed with the aerosol and water ice abundance profiles, optical properties, and size distributions of the test model (see Sec. 4, Fig. 2, and Table 1). 

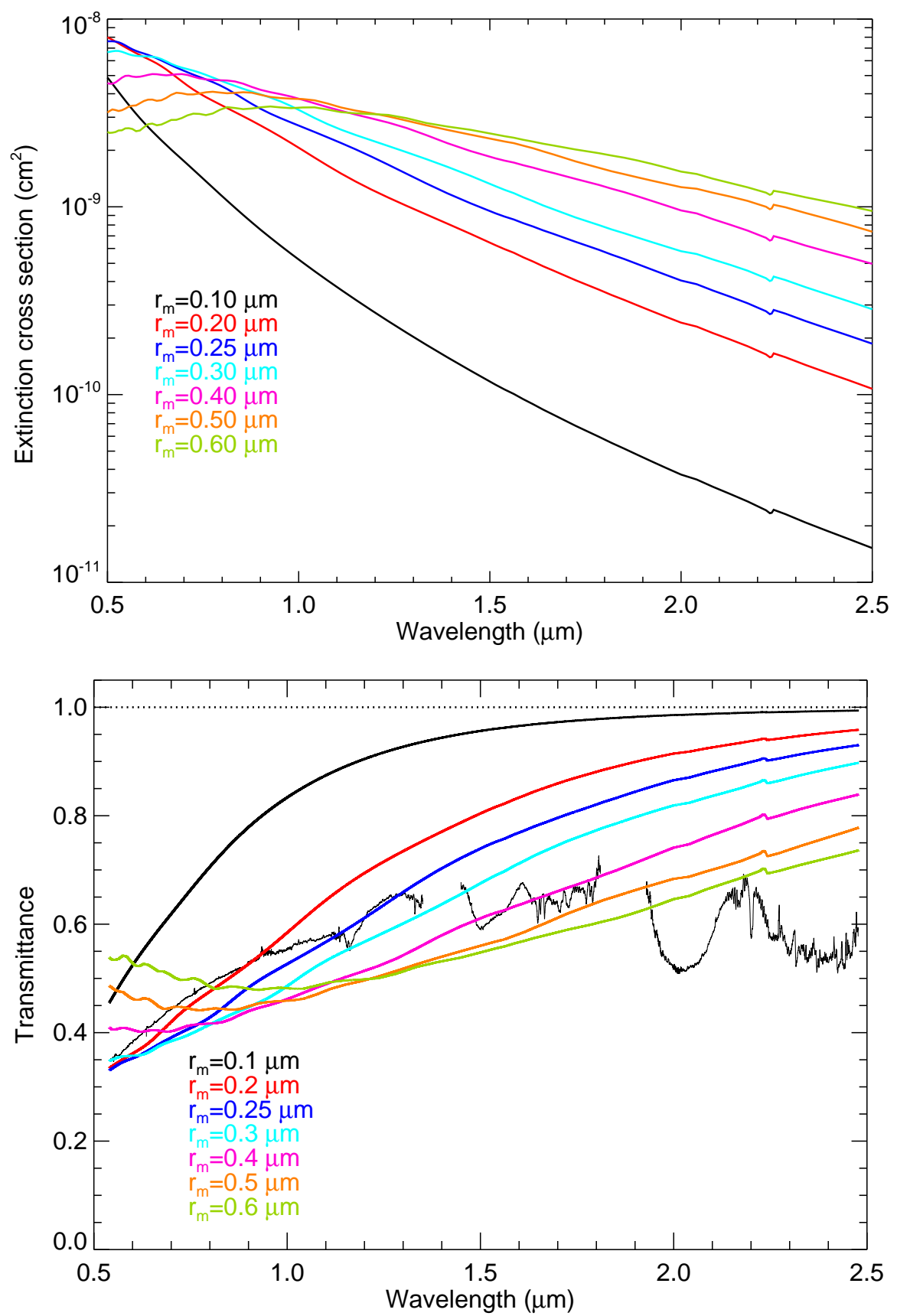

Figure 5. Effect of aerosol particle size on the transmission of the early-umbra spectrum (see Table 1). Top: the haze extinction for particle size in the range of 0.1 to $0.6 \mu \mathrm{m}$. The cross section has been scaled to the volume of the $r_{m}=0.25 \mu \mathrm{m}$ particles. Bottom: The simulated transmissions for the limb geometry of the early umbra spectrum. The measured early umbra spectrum (broken black solid line) is plotted for reference. 

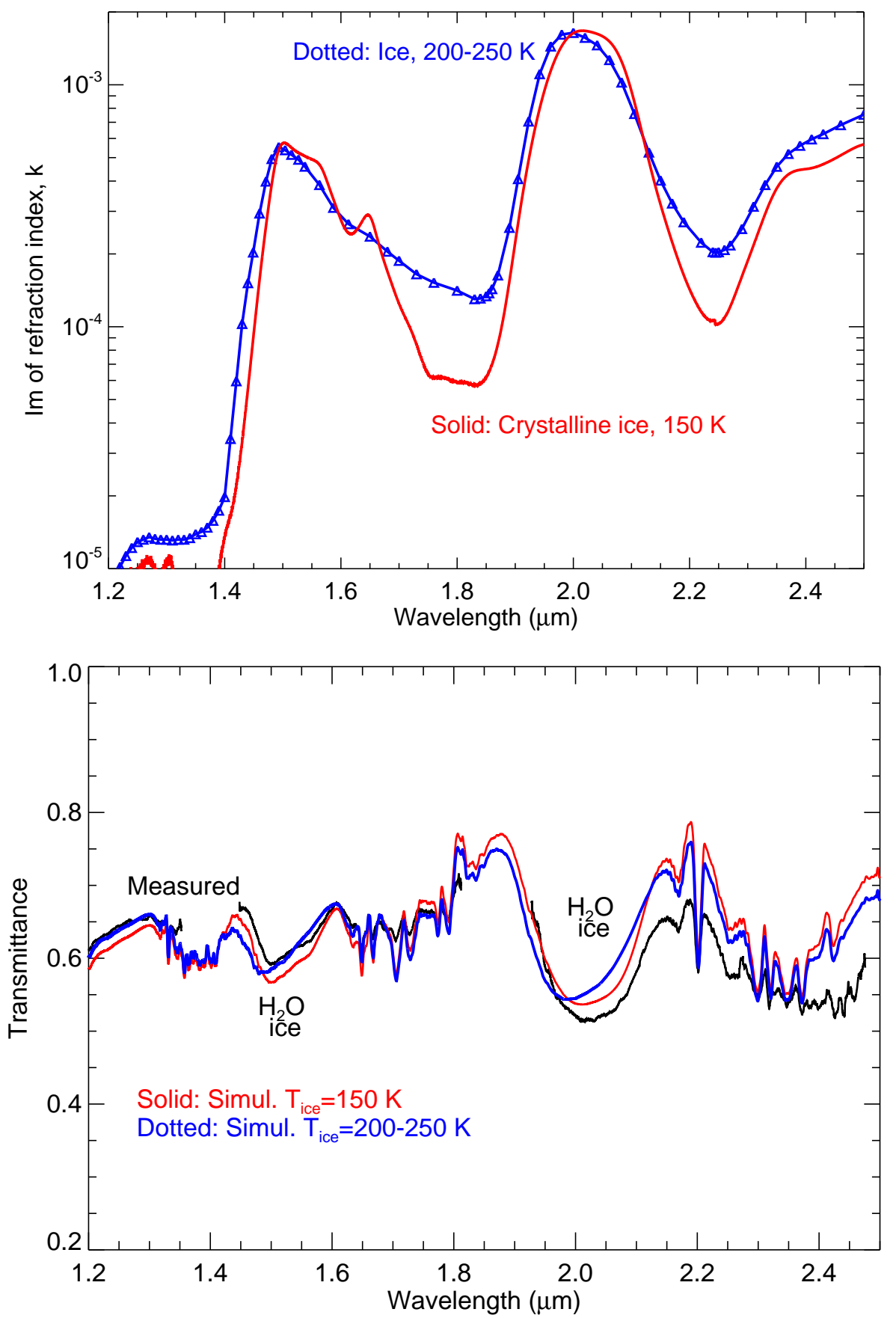

Figure 6. Effects of the temperature of the water ice on the simulated spectra for the early umbra conditions (see Table 1). Top: $k$ index; bottom: simulated and measured (early umbra) transmission spectra. Note that the crystalline ice at a temperature of $150 \mathrm{~K}$ (Mastrapa et al. 2008) has a better fit at the peaks of the ice absorption bands near 1.5 and $2.0 \mu \mathrm{m}$, while for the ice at a temperature of 200-250 K (Warren \& Brandt 2008) the peaks are shifted to shorter wavelengths. 

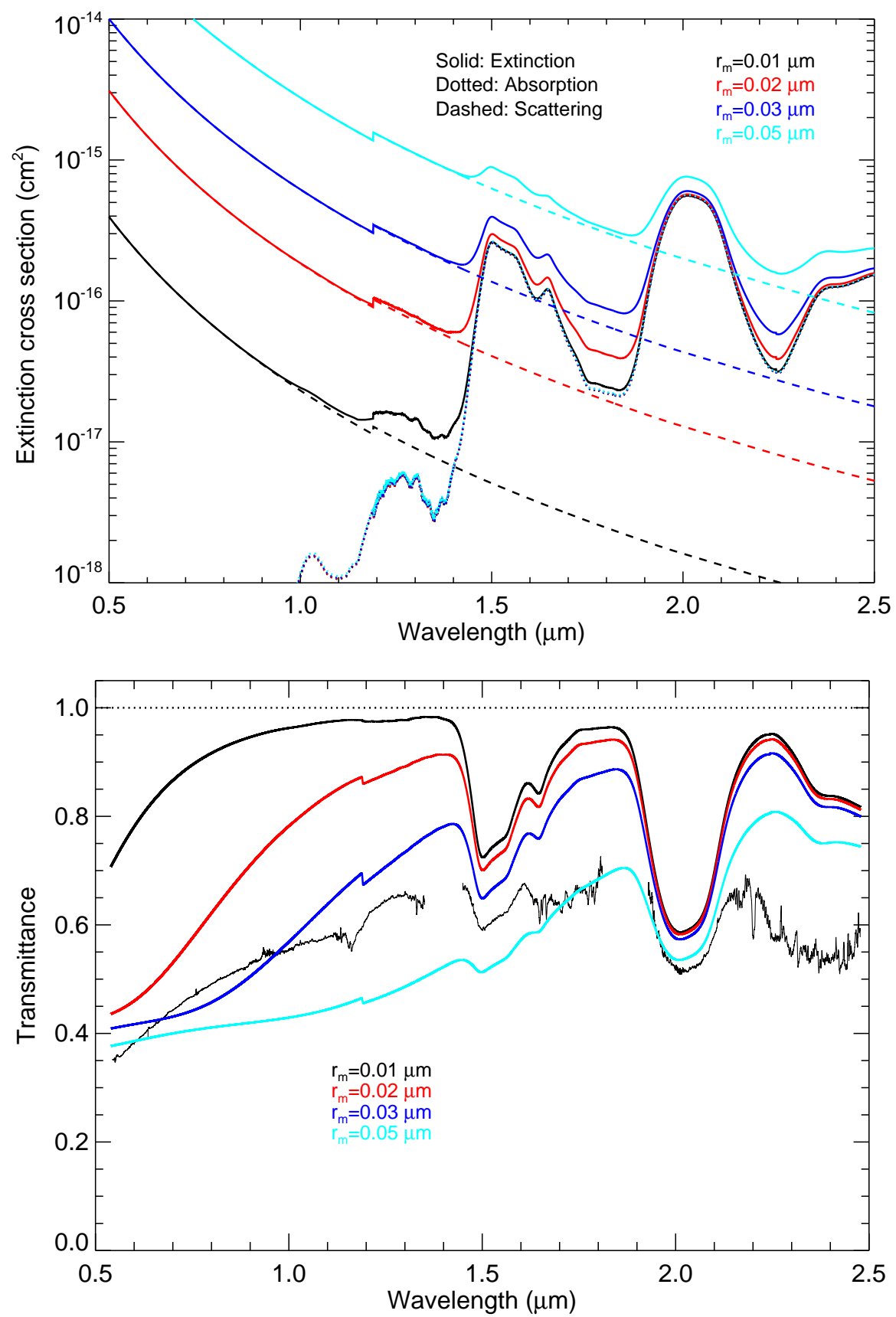

Figure 7. Effects of the water ice particles' size on the simulated spectra for the early umbra conditions for the test model conditions (see Table 1). Top panel: extinction cross sections scaled to the volume of the particles with a mean radius of $0.01 \mu \mathrm{m}$. Bottom: The simulated transmissions for the geometry of the early umbra spectrum. The same ice particle volume (mass), that of the test model, was assumed in all cases. The measured early umbra spectrum (broken black solid line) is plotted for reference. 


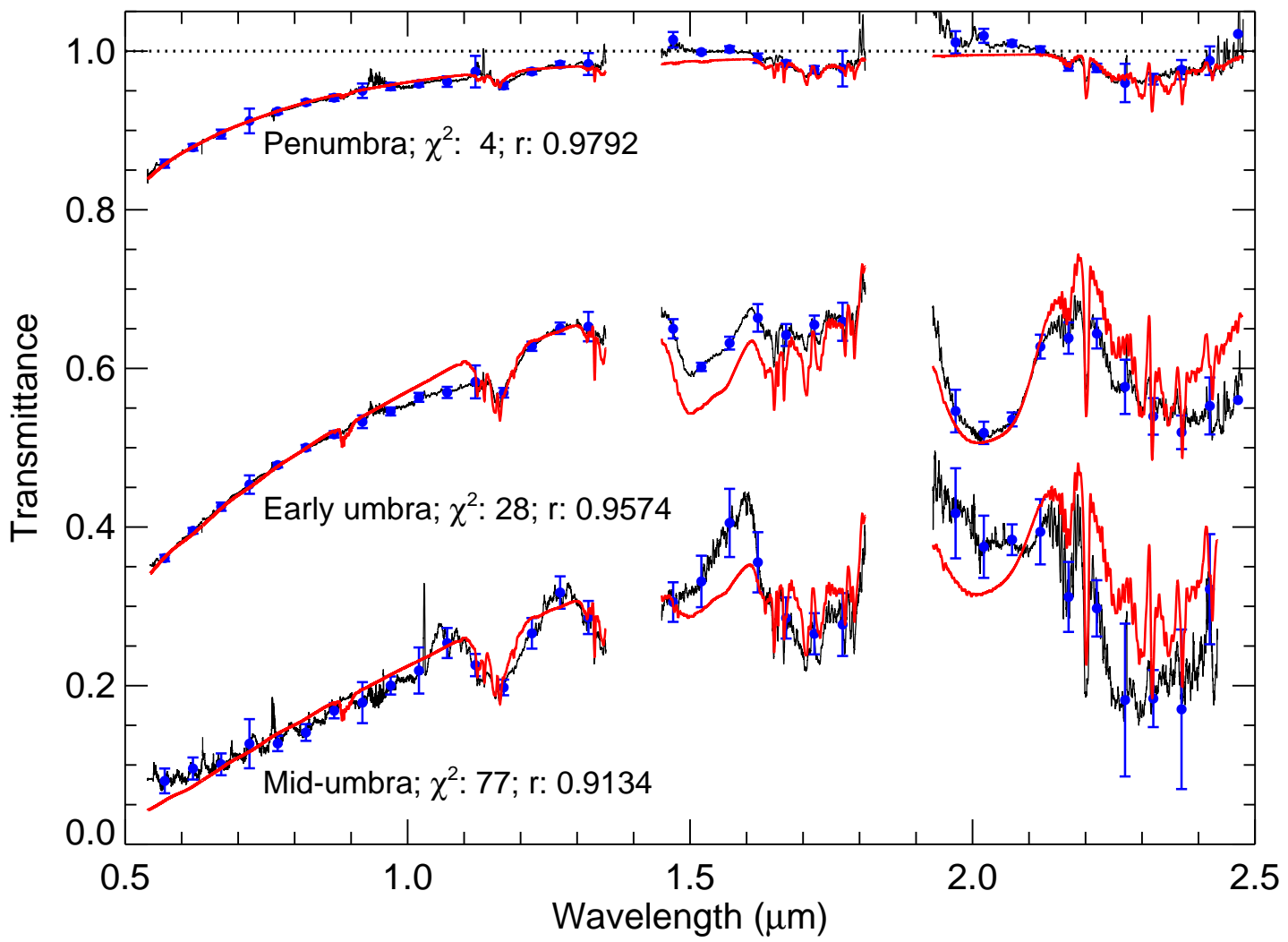

Figure 8. The measured and the best-fit spectra for the penumbra conditions (top), the early phase of the umbra (middle) and mid-umbra (bottom). Measured transmission spectra in black and simulations in red. The blue dots and the vertical bars denote the estimated noise error of the transmission spectra. Values of the $\chi^{2}$ and the correlation coefficients are also given for each fit. The number of spectral points in each case are 44272, 44012, and 43509, respectively.

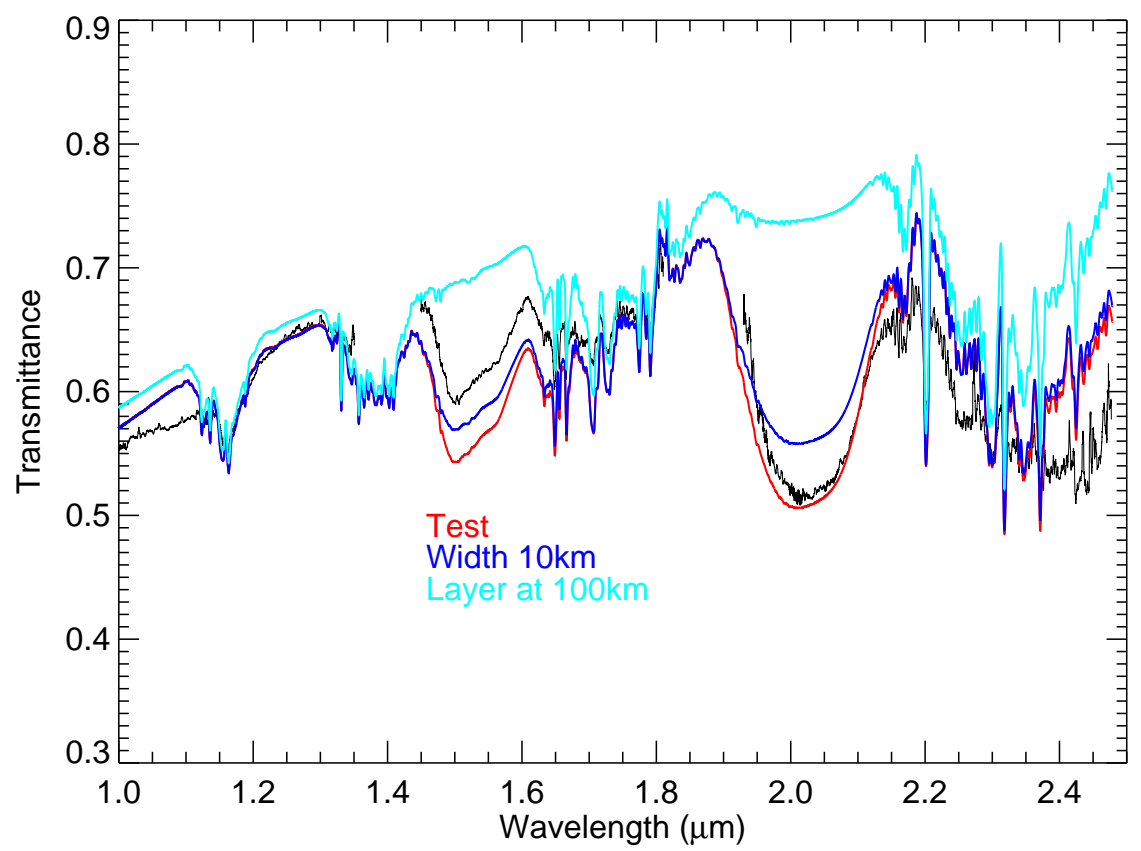

Figure 9. Simulations varying the extension of the water ice cloud (compressing it to $10 \mathrm{~km}$ wide at $170 \mathrm{~km}$, keeping the same column content) (dark blue), and by varying the height of the water ice cloud down to $100 \mathrm{~km}$ (turquoise) for the umbra conditions (see Table 1). The observed (black) and the best fit (red) are shown for comparison. 


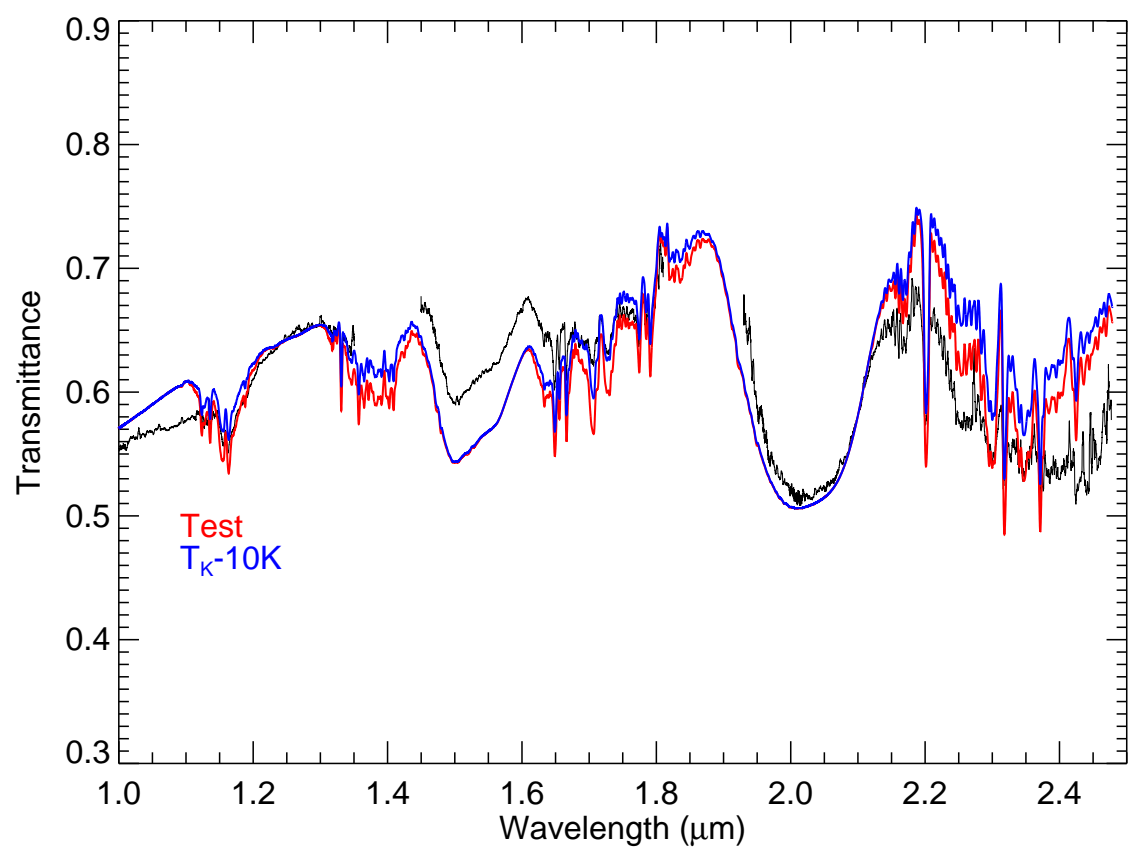

Figure 10. Effects of decreasing the kinetic temperature profile by $10 \mathrm{~K}$. Red curve: transmission with the unperturbed kinetic temperature profile; blue: with $T_{k}$ decreased by $10 \mathrm{~K}$ (see Table 1 ). The observed transmission (black broken solid line) is also shown for reference.

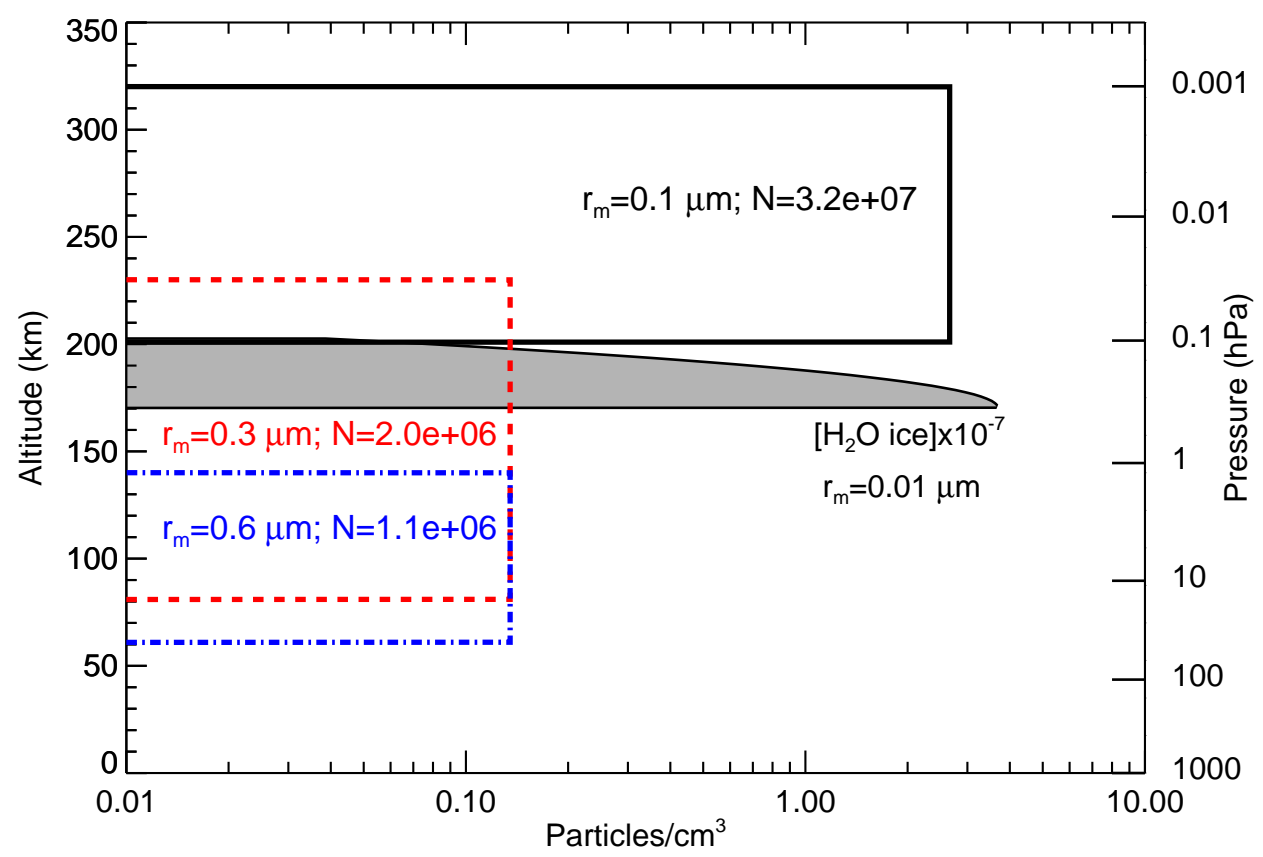

Figure 11. The distribution of haze (aerosols) concentrations (rectangles) and of the water ice layer (shaded area) derived from the spectra. $N$ is the column density of the aerosols in particles $/ \mathrm{cm}^{2}$. 
Table 1. Parameters of the models used in the sensitivity study.

\begin{tabular}{lcccccc}
\hline \hline Model & $\begin{array}{c}\text { Aerosol } \\
\text { particle size } \\
(\mu \mathrm{m})\end{array}$ & $\begin{array}{c}\text { Water ice } \\
\text { temp. } \\
(\mathrm{K})\end{array}$ & $\begin{array}{c}\text { Water ice } \\
\text { particle size } \\
(\mu \mathrm{m})\end{array}$ & $\begin{array}{c}\text { Water ice } \\
\text { layer width } \\
(\mathrm{km})\end{array}$ & $\begin{array}{c}\text { Water ice } \\
\text { layer height } \\
(\mathrm{km})\end{array}$ & $\begin{array}{c}\text { Temp. } \\
\text { profile }\end{array}$ \\
\hline Test (nominal) & 0.25 & 150 & 0.01 & 30 & 170 & Nominal (Fig. 2) \\
A (Fig. 5) & $0.1-0.6$ & 150 & 0.01 & 30 & 170 & Nominal (Fig. 2) \\
B (Fig. 6) & 0.25 & $200-250$ & 0.01 & 30 & 170 & Nominal (Fig. 2) \\
C (Fig. 7) & 0.25 & 150 & $0.01-0.05$ & 30 & 170 & Nominal (Fig. 2) \\
D (Fig. 9a) & 0.25 & 150 & 0.01 & 10 & 170 & Nominal (Fig. 2) \\
E (Fig. 9b) & 0.25 & 150 & 0.01 & 30 & 100 & Nominal (Fig. 2) \\
F (Fig. 10) & 0.25 & 150 & 0.01 & 30 & 170 & Reduced by 10 K \\
\hline
\end{tabular}

